\title{
Les soins en optométrie au patient atteint de diabète 2008: Un document central de l'Association canadienne des optométristes
}

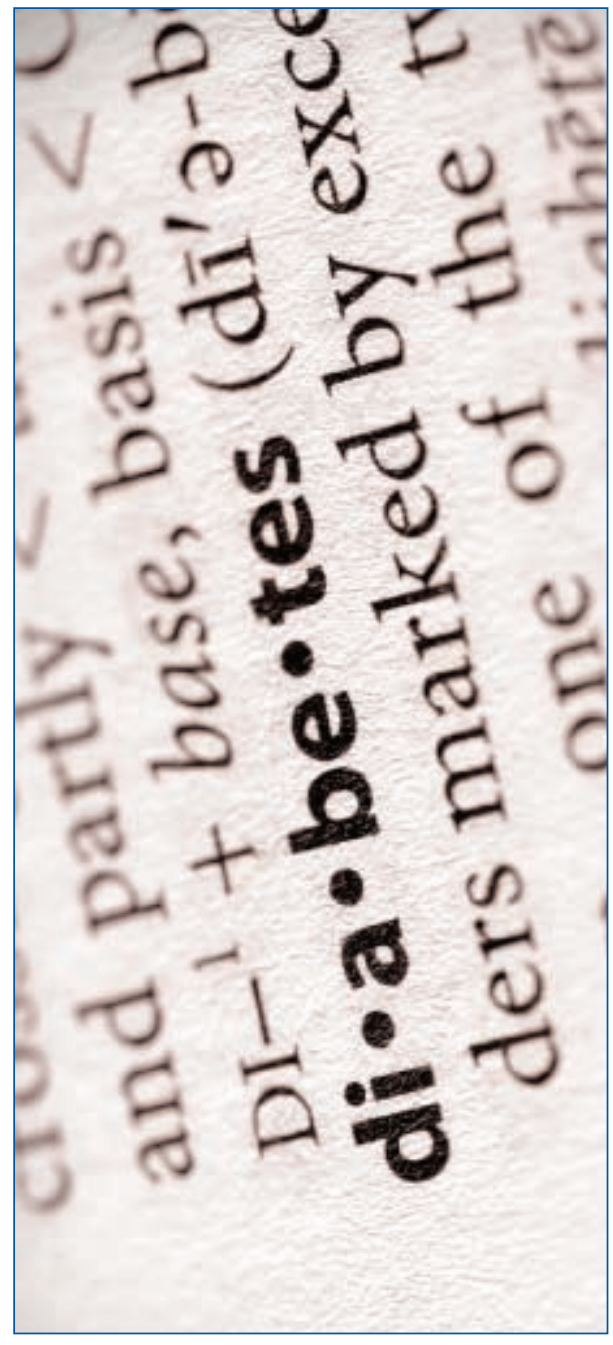

Introduction

The objective of this document is to provide the reader with an overview of the epidemic of diabetes currently facing Canada and the morbidity and mortality associated with this growing healthcare burden.

Specifically, an evidence based, patient centered, cost effective role of the optometrist in the eye care of Canadians with diabetes will be presented.

\section{Diabetes in Canada}

The current number of people living with diabetes in Canada is approximately two million ${ }^{1}$. Type 2 diabetes is the most common form of the disease, accounting for $90 \%$ of cases. The prevalence of type 2 diabetes is increasing dramatically with current estimates confidently predicting a doubling of cases by $2025^{2,3}$. In addition, the prevalence of diabetes in First Nations people in Canada is substantially higher than in the general population ${ }^{4}$. The "diabetes epidemic" is projected to increase the total number of cases of diabetes in Canada to three million by the end of this decade. Diabetes represents a potential doomsday threat to human health in the $21^{\text {st }}$ century ${ }^{5,6}$. It has been estimated that diabetes is a contributing factor in the
Dr Richard Lee, BSC, OD

Dr Garry Best, OD

Dr Michael Dennis, BSc, OD

Dr Henry Smit, OD

Dr Chris Hudson, PhD, MCOptom, FAAO 


\section{L'ACTUALITÉ FEATURE ARTICLE}

The following Flow chart should be used by optometrists to manage patients with Diabetes and inform other members of the Diabetic Healthcare Team (DHC Team) of their findings.

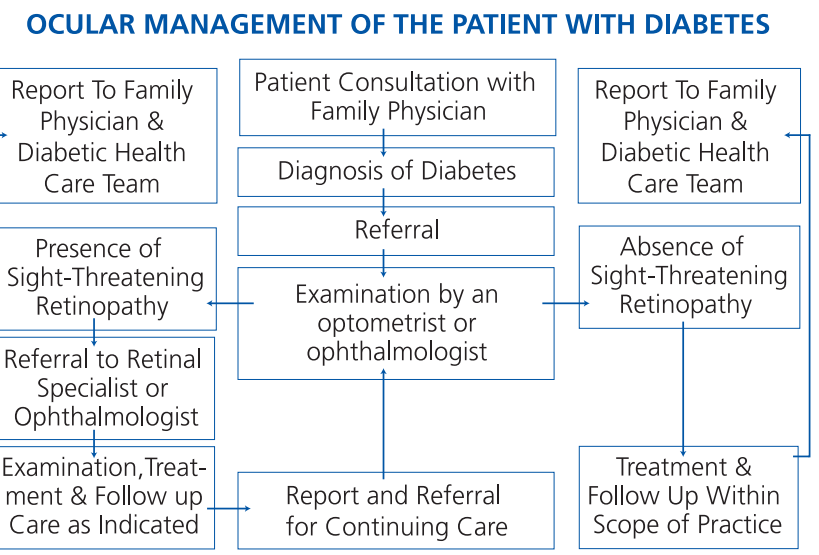

Note: Diabetic Health Care Team (DHC Team) refers to the core team which includes the Physician (family physician and/or specialist) and the Diabetic Educators (Nurse and Dietician).

\section{Introduction}

Ce document a pour but de présenter une vue d'ensemble de l'épidémie de diabète à laquelle fait actuellement face le Canada, ainsi que de la morbidité et de la mortalité associées à ce fardeau croissant des soins de santé.

On y présentera spécifiquementlerôle del'optométriste dans les soins oculovisuels des Canadiens diabétiques, rôle rentable, axé sur le patient et basé sur des résultats cliniques et scientifiques.

\section{Le diabète au Canada}

Il y a actuellement environ deux millions ${ }^{1}$ de personnes diabétiques au Canada. Le diabète de type 2 est la forme la plus courante de la maladie, représentant $90 \%$ des cas. La prévalence du diabète de type 2 augmente en flèche, les estimations actuelles prévoyant avec certitude deux fois plus de cas d'ici $2025^{2,3}$. De plus, la prévalence du diabète chez les peuples des Premières nations au Canada est considérablement plus élevée que dans la population générale 4 . On prévoit que cette "épidémie diabétique» fera passer le nombre total de cas de diabète au Canada à trois millions à la fin de cette décennie. Le diabète représente une menace apocalyptique potentielle à la santé humaine au $\mathrm{XXI}^{\mathrm{e}}$ siècle ${ }^{5,6}$. On estime que le diabète est un facteur contribuant au décès de $41500 \mathrm{Ca}$ nadiens par année, et ce nombre ira certainement en augmentant ${ }^{7}$.

Au niveau de la morbidité, le diabète a une incidence sur presque tous les organes du corps. Le diabète est fortement associé à l'hypertension, à la dyslipidémie et à l'obésité. Le diabète double le risque d'accident vasculaire cérébral, cause 33\% des nouveaux cas de néphropathie au stade terminal, quadruple le risque de cardiopathie et est la cause première des amputations des membres inférieurs non traumatiques; il est aussi la cause première de la cécité chez les adultes âgés de 25 à 75 ans et peut aussi causer la neuropathie diabétique ${ }^{8}$, une forme douloureuse de détérioration des nerfs. Les statistiques révèlent que l'espérance de vie d'une personne atteinte du diabète de type 2 peut être abrégée de cinq à dix ans ${ }^{9}$.

L'augmentation prévue du diabète de type 2 pendant les deux prochaines décennies aura une incidence sans précédent sur les services de soins de santé dispensés aux Canadiens. En 2003, Santé Canada ${ }^{10}$ a estimé qu'on dépensait annuellement au moins 13,5 milliards de dollars pour le traitement du diabète et des complications diabétiques. L'American Diabetes Association a nettement préparé le terrain pour l'intégration des professionnels des soins de santé lorsqu'elle a affirmé qu'il est temps que les professionnels de la santé traitent le diabète de façon vigoureuse, que les patients s'occupent de leur diabète avec le plus grand sérieux et que le système de soins de santé fournisse les ressources nécessaires pour que les deux parties soient gagnantes ${ }^{11}$. Les intervenants, dont l'Association médicale canadienne, l'Association canadienne du diabète, la Société canadienne d'ophtalmologie et l'ACO, ont déclaré que les professionnels de la santé doivent élaborer un système intégré qui garantisse une approche des soins diabétiques d'une qualité exceptionnelle $\mathrm{e}^{12,13}$.

\section{Types de diabète}

\section{Diabète de type 1}

Ce type de diabète constitue environ $10 \%$ des cas de diabète. Le diabète de type 1 se caractérise par une destruction d'origine auto-immune des cellules $\beta$ du pancréas. Cette atteinte conduit habituellement à une 


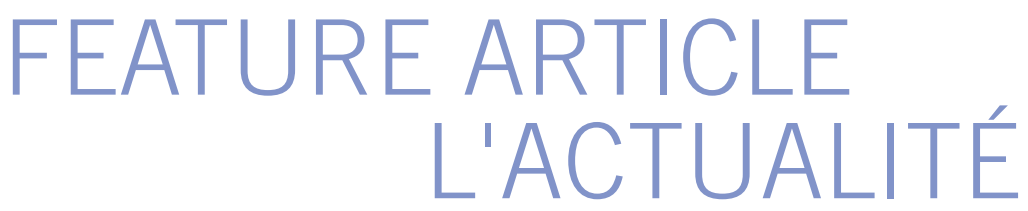

déficience insulinique totale. Il apparaît généralement de façon subite chez des jeunes gens ayant souvent des antécédents d'infections, en particulier d'origine virale. Même si on ne considère plus le diabète de type 1 comme une maladie spécifique aux jeunes gens ${ }^{14}$, la manifestation initiale de la maladie survient souvent chez un enfant qui peut présenter une gamme de symptômes allant des symptômes légers, non spécifiques jusqu'au coma ${ }^{15}$. Les personnes chez qui on a diagnostiqué récemment un diabète de type 1 peuvent présenter les symptômes suivants: perte de poids, léthargie, polyurie, polydipsie, perte d'appétit, nausées, vomissements, vision floue et état mental perturbé ${ }^{16}$. La polyurie, la polydipsie et la vision floue sont le résultat d'un état hyperosmolaire, tandis que la perte de poids est la conséquence d'une transformation des acides aminés en glucose et cétones. Les complications aiguës du diabète incluent le coma hypoglycémique, l'hypoglycémie, l'acidocétose (ACD) et le syndrome non cétosique hyperosmolaire $(\mathrm{SNCH})$. L'ACD est une manifestation commune de cette maladie et comporte un risque accru d'œdème cérébral $(\mathrm{OC})^{17}$. Les taux de mortalité de l'ACD se situent entre $0,15 \%$ et $0,31 \%$, et l'OC constitue environ $57 \%$ à $87 \%$ de ces décès ${ }^{18,19}$. Les patients diabétiques souffrant d'ACD auront souvent une haleine cétonique.

La pathophysiologie du diabète de type 1 serait un processus auto-immun dans lequel les cellules bêta du pancréas, lesquelles produisent l'insuline, sont détruites ${ }^{20}$. Le processus auto-immunitaire peut être déclenché par une réaction à une infection comme la rubéole et le cytomégalovirus, ou même par l'administration d'un vaccin. On ne comprend cependant pas bien ce processus puisqu'il y a peu de preuves scientifiques et cliniques à l'appui. De plus, la réponse auto-immune peut être influencée par des anticorps luttant contre des protéines du lait de vache. En effet, les preuves cliniques et scientifiques appuyant le rôle des facteurs environnementaux sont tellement faibles que certains affirment qu'ils ne serviraient qu'à modifier la pathogenèse de la maladie plutôt qu'à agir comme déclencheurs. On a aussi avancé l'idée qu'une vulnérabilité génétique pouvait développer un diabète de type 1.

L'insuline agit comme un point de passage qui permet au glucose de traverser les membranes cellulaires des tissus insulino-dépendants, principalement les tissus adipeux et musculaires. Ces tissus doivent alors utiliser comme sources d'énergie d'autres substrats comme les acides gras libres, ce qui provoque la formation de cétones et éventuellement l'ACD. Les tissus non insulinodépendants comme le cerveau, le système nerveux, le cortex du rein et la moelle épinière deviennent submergés par l'accroissement des niveaux de glucose dans le sang et sont endommagés par la formation de produits terminaux avancés de glycation/glycatés (PTAG). De plus, la déviation du métabolisme du glucose vers le sorbitol provoque une production excessive de sorbitol et de fructose et donc un stress osmotique. L'hyperglycémie chronique donne lieu à une quantité de changements métaboliques supplémentaires qui endommagent encore plus les tissus: anomalies au métabolisme des lipides, détérioration oxydative accrue, hyperinsulinémie, hyperperfusion, hyperviscosité, désordre plaquettaire et activation des facteurs de croissance. Ces réactions provoquent des cataractes et des maladies microvasculaires des yeux (glaucome néovasculaire et rétinopathie), des reins (néphropathie) et des nerfs (neuropathie).

Les diabétiques de type 1 présentent rarement des complications oculaires avant au moins cinq ans après le diagnostic. Après 10 ans de vie diabétique, $60 \%$ de ces personnes manifesteront quelques signes de rétinopathie diabétique et, après 15 ans, il est vraisemblable que tous les diabétiques de type 1 seront atteints de rétinopathie diabétique. Après 15 ans, $42 \%$ des diabétiques de type 1 développeront un œè̀me maculaire diabétique $(\mathrm{OMD})^{21}$. À la quinzième année, la rétinopathie diabétique deviendra proliférante chez $23 \%$ d'entre eux, et après 20 ans, chez $50 \%$ d'entre eux $x^{22,23,24}$.

\section{Diabète de type 2}

L'incidence du diabète de type 2 augmente significativement avec l'âge, se manifestant typiquement au mitan de la vie ou plus tard, et même actuellement chez l'enfant/ adolescent. Environ $90 \%$ des cas de diabète sont de type 2 . Le diabète de type 2 se caractérise par une combinaison de résistance à l'insuline et d'une déficience de la sécrétion d'insuline (épuisement des cellules-ß). Cette maladie se développe généralement de façon lente, le premier signe étant une résistance à l'insuline périphérique. Le pancréas répond en augmentant sa

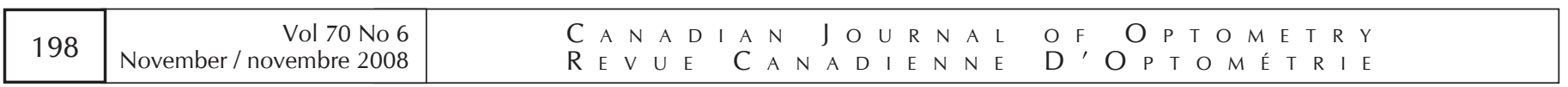




\section{L'ACTUALITÉ FEATURE ARTICLE}

production d'insuline, ce qui conduità l'hyperinsulinémie. Les niveaux de glucose peuvent se maintenir dans le sang près de la normale pendant des années, avant que surviennent l'intolérance au glucose et la diminution des fonctions pancréatiques. Éventuellement, la teneur en glucose dans le sang augmente tout comme le risque de maladie microvasculaire. Tout comme le diabète de type 1, la pathophysiologie du diabète de type 2 est plurifactorielle et n'est pas totalement comprise. On croit fortement qu'il est lié à une prédisposition génétique de même qu'au changement de style de vie au cours du siècle précédent, passant de la «famine au festin », lié à une diminution de l'activité physique ${ }^{6}$. Le tableau clinique du diabète de type 2 est aussi mieux compris. Particulièrement, il semble y avoir un lien entre le diabète de type 2 et la maladie mentale, spécialement avec la dépression et l'anxiété. Il y a deux fois plus de risque de dépression chez les diabétiques, et il y a une association entre la dépression, l'hyperglycémie et le risque de complication diabétique $e^{25}$. De plus, la prévalence du diabète de type 2 est beaucoup plus élevée chez les patients souffrant de schizophrénie, de troubles bipolaires ou d'un trouble dépressif grave ${ }^{26}$; on va même jusqu'à dire que la schizophrénie est maintenant un facteur de risque reconnu dans le développement du diabète de type 2 .

Le diabète de type 2 en particulier tout comme la maladie cardiovasculaire et l'accident vasculaire cérébral sont souvent précédés par un syndrome métabolique. Le syndrome métabolique est un ensemble de facteurs de risques métaboliques qui surviennent simultanément chez la personne atteinte. Ces facteurs de risque incluent : résistance à l'insuline, hyperglycémie, obésité, hypertension et dyslipidémie (augmentation des lipoprotéines de basse densité ou LDL, diminution des lipoprotéines de haute densité ou HDL, ce qui cause une augmentation des anomalies dans la coagulation du sang). Il s'ensuit que beaucoup de diabétiques, spécialement de type 2 , souffrent aussi d'hypertension et d'anomalies des lipides de façon concomitante, ce qui produit les signes rétiniens caractéristiques du croisement artério-veineux et des exsudats secs de même que les signes de la rétinopathie diabétique. De $20 \%$ à $30 \%$ des personnes vivant dans les pays industrialisés sont susceptibles d'être atteintes du syndrome métabolique. La prévalence augmente à
$60 \%$ chez les personnes obèses.

On rapporte qu'entre $80 \%$ et $90 \%$ des diabétiques de type 2 nouvellement diagnostiqués sont des personnes obèses. Cliniquement l'obésité se définit comme un indice de masse corporelle (IMC) supérieur à $30^{27}$. Il y a une épidémie d'obésité au Canada, où les taux d'obésité chez les enfants se sont accrus dans les 15 dernières années, passant de $2 \%$ à $10 \%$ chez les garçons et de $2 \%$ à $9 \%$ chez les filles ${ }^{28}$. De 1980 à 2000, l'obésité chez les adultes a doublé ${ }^{2}$. Les autres facteurs de risque du diabète de type 2 incluent : poids à la naissance de plus de neuf livres, antécédents de diabète gestationnel, tabagisme, autres maladies vasculaires et antécédents d'intolérance au glucose ${ }^{30}$. Il a été démontré que $20 \%$ des diabétiques de type 2 présenteront une certaine forme de rétinopathie diabétique au moment du diagnostic. À la quinzième année, de $60 \%$ à $85 \%$ des diabétiques présenteront des signes de rétinopathie diabétique. Seulement $4 \%$ d'entre eux développeront la RDP (rétinopathie diabétique proliférante) dans les quatre années suivant le diagnostic et, après 15 ans, de $5 \%$ à $20 \%$ d'entre eux développeront la RDP. Après 15 ans, $80 \%$ des diabétiques de type 2 développeront un $\mathrm{OMD}^{21}$.

\section{Diabète gestationnel}

Ce type de diabète survient dans environ $5 \%$ de toutes les grossesses. Le diabète gestationnel augmente le risque d'hypertension et de césarienne. Le risque de mortinatalité chez les femmes diabétiques enceintes est presque deux fois plus élevé que chez les femmes enceintes non diabétiques ${ }^{31}$. Le diabète gestationnel est associé à l'augmentation de la circonférence abdominale fœtale et à la macrosomie fœtale; il semble que les rejetons des femmes souffrant de diabète gestationnel sont plus à risque de souffrir d'obésité et ont une prévalence plus élevée d'être atteints du diabète de type 2 durant leur vie ${ }^{32}$. Le diabète gestationnel qui survient durant la grossesse n'a pas été associé avec le développement de la rétinopathie diabétique durant cette grossesse.

\section{Traitement}

Traitement du diabète de type 1

Le diabétique de type 1 doit utiliser l'insuline exogène pour vivre, habituellement injectée par voie sous-cutanée. D’autres méthodes d'apport de l'insuline
C A N A D I A N J O U R N A L OF OP T O METR Y

$R$ E V U E C A N A D I E N N E D ' O P T O M É T R I E
Vol 70 No 6

November / novembre 2008 


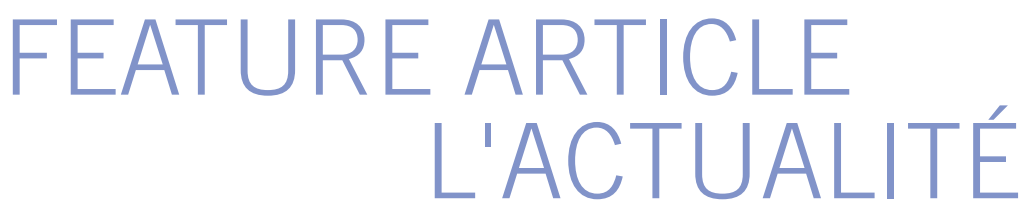

sont aussi reconnues ou encore à diverses étapes de développement (pompe, voie orale, nasale, transdermique et inhalation). Les éducateurs spécialisés en diabète enseignent aux diabétiques comment doser la quantité et le type d'insuline en fonction du glucose dans le sang et de l'apport calorique. Des types variés d'insuline ayant des modes d'action différents (action rapide, action à court et long termes) sont maintenant disponibles et peuvent être utilisés en combinaison pour obtenir des fluctuations diurnes améliorées et un contrôle glycémique à long terme plus précis.

\section{Traitement du diabète de type 2}

Le régime alimentaire et l'exercice physique demeurent les pierres d'assise du traitement du diabète de type 2 , au même titre qu'un contrôle approprié de la tension artérielle et un traitement de la dyslipidémie. La diminution de l'apport calorique provoque une perte moyenne de $5 \%$ du poids corporel initial; il y a aussi une diminution associée de la progression du diabète de type 2 de $58 \%$ chez les personnes souffrant d'intolérance au glucose $^{33}$. Les lignes directrices de la pratique clinique de l'Association canadienne du diabète recommandent que les diabétiques de type 2 fassent au moins chaque semaine 150 minutes d'exercices aérobiques modérés, répartis sur trois jours non consécutifs, ou quatre heures ou plus d'exercices par semaine ${ }^{34}$.

$\mathrm{Si}$ le régime alimentaire et l'exercice physique ne permettent pas à la personne d'atteindre ses cibles de glucose dans le sang, on recommande alors la médication. Le médicament de premier choix est un biguanide (Metformin). Il diminue la quantité de glucose formée dans le foie et facilite le passage de ce glucose dans les cellules du corps par la circulation sanguine. Les agents sensibilisateurs de l'insuline, rosiglitazone (Avandia) et pioglitazone (Actos) constituent une deuxième option. Ces médicaments permettent à l'organisme d'être plus réceptif à sa propre insuline. Le troisième choix sont les sécrétagogues de l'insuline qui se classent en deux catégories : les sulfonylurés [glyburide (DiaBeta), glicazide (Diamicron et DiamicronMR) et glimépiride (Amaryl)]; et les nonsulfonylurés; [répaglinide (Gluconorm) et natéglinide (Starlix)]. Ces médicaments stimulent le pancréas à produire de l'insuline. Aussi, les inhibiteurs des alpha-glucosidases (Prandase) bloquent dans l'intestin une enzyme qui transforme des hydrates de carbone complexes en glucose. L'inhibiteur du dipeptidylpeptidase 4 (DPP-4), la sitagliptine (Januvia), est un produit récent qui stimule simultanément la sécrétion d'insuline et empêche la production de glucagon par le pancréas (diminuant ainsi la quantité de glucose produite dans le sang par le foie).

Quel que soit le type de diabète, la façon clé de gérer cet état est de maintenir le glucose sanguin, la tension artérielle et le cholestérol dans des limites acceptables. De plus, il est essentiel d'y associer un régime alimentaire, une gestion du poids et une activité physique accrue, de même que des soins oculovisuels et podologiques appropriés, etc.

\section{La perte de la vision et le vieillissement}

Les Canadiens redoutent la perte de la vue plus que la perte de tout autre sens. La perte de vision a une incidence énorme sur la qualité de vie et est extrêmement coûteuse dans une perspective sociétale et économique (www.costofblindness.org). On prévoit que le vieillissement de la population canadienne aura des conséquences désastreuses sur les niveaux de perte de vision au moment où les baby-boomers atteindront 60 ans $^{35,36}$. À partir de 65 ans, une personne sur neuf connaitra une perte de vision grave, et à partir de 75 ans, cela passera à une personne sur quatre ${ }^{37}$; ces tendances sont encore plus accentuées chez les peuples des Premières nations.

\section{Le diabète et l'œil}

L'effet du diabète sur l'œil et la vision varie, allant de la vision floue légère et passagère jusqu'à la cécité. Les diabétiques courent un risque accru de paralysie des muscles de l'orbite, le plus souvent des nerfs crâniens (le 3 , le 6 et rarement le 4). Les diabétiques développent des cataractes plus tôt que la population générale ${ }^{38}$. Le glaucome néovasculaire, que l'on croit être causé par le même mécanisme que la RDP, est un type de glaucome très difficile à traiter et son pronostic d'amélioration est faible. La rétinopathie diabétique, maladie microvasculaire de la rétine, demeure la complication diabétique visuelle la plus sérieuse. La rétinopathie diabétique est l'une des principales causes des handicaps visuels et de la cécité chez les Canadiens âgés de 30 à 69 ans ${ }^{39}$. Les diabétiques sont 25 fois plus susceptibles que la population générale

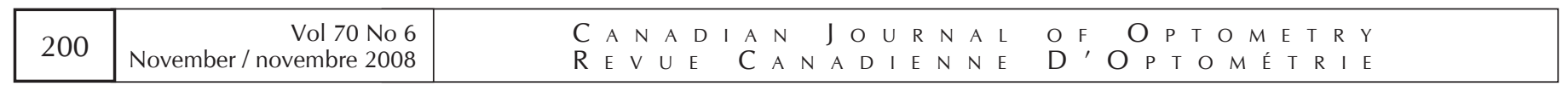




\section{L'ACTUALITÉ FEATURE ARTICLE}

de devenir aveugles ${ }^{40}$. La perte de la vue associée à la rétinopathie diabétique est causée par l'œdème maculaire diabétique (OMD)/ maculopathie et par les séquelles de la RDP, à savoir le décollement rétinien par traction et l'hémorragie du vitré. L'OMD cause habituellement une perte progressive de la vue, mais représente la cause la plus commune des handicaps visuels et de la cécité chez les diabétiques, tandis que la RDP provoque une perte de vision grave soudaine (AV $=5 / 200)$. Les changements vasculaires qui surviennent dans l'œil permettent de prévoir des changements vasculaires dans d'autres parties du corps ${ }^{41,42}$. Un examen oculovisuel complet par un optométriste peut permettre de déceler les complications du diabète qui sont menaçantes pour la vue; environ $50 \%$ de la population vivent avec un diabète non encore diagnostiqué.

\section{La rétine}

La rétine est une membrane nerveuse semi-transparente d'une épaisseur d'environ 200 micromètres (environ l'épaisseur d'un ongle de doigt). Tapissant le fond de l'œil, elle est située au carrefour de deux circulations sanguines: la circulation choroïdienne derrière la rétine et la circulation rétinienne sur la surface frontale de la rétine. Ces deux circulations sanguines proviennent de l'artère ophtalmique qui est le premier embranchement de l'artère carotide interne. La rétine n'est pas un tissu insulinodépendant, ce qui signifie que le glucose pénètre facilement les cellules rétiniennes sans avoir besoin d'insuline. Cependant, un niveau de glucose sanguin trop élevé peut causer une toxicité au tissu rétinien.

\section{Pathophysiologie de la rétinopathie diabétique}

La rétinopathie diabétique se caractérise par l'instabilité, la décompensation et l'effondrement du réseau microvasculaire rétinien ${ }^{43}$. Morphologiquement, ces changements microvasculaires causent un durcissement de la membrane de de base des capillaires, une perte de péricytes, une déplétion lente des cellules musculaires, une perte des cellules endothéliales vasculaires, une occlusion et une recanalisation vasculaires et, à la limite, une néovascularisation. La détérioration des vaisseaux rétiniens provoque la fermeture des lits capillaires et conduit à l'œdème et à l'ischémie.
On croit que le facteur de croissance vasculaire endothélial (VEGF), une cytokine produite par les cellules vasculaires rétiniennes, contrôle la perméabilité vasculaire en cas d'hyperglycémie, et provoque l'angiogénèse à la suite d'une hypoxie. Dans le cas d'un OMD, le VEGF et les produits terminaux avancés de glycation devraient, selon les prédictions, réguler à la hausse le niveau des molécules d'adhérence intercellulaire (ICAM1) ce qui, à son tour, augmente la leucostase dans les vaisseaux rétiniens, accroît la perméabilité vasculaire et diminue la perfusion capillaire. Dans le cas de la RDP, comme il y a un accroissement des niveaux du VEGF en réponse à l'hypoxie, le facteur antiangiogénique dérivé de l'épithélium pigmentaire serait à la baisse, créant un environnement pro-angiogénique.

Cliniquement, la rétinopathie diabétique peut être classée d'une façon sommaire comme non proliférante et non présente (RDNP), comme proliférante (RDP), et/ ou comme odème maculaire diabétique cliniquement significatif (OMDCS). La RDNP se subdivise en trois catégories: légère à modérée, modérée à sévère, sévère et très sévère.

Femmes enceintes et diabète préexistant

Chez les femmes ayant un diabète préexistant, la rétinopathie risque de progresser rapidement. Toutefois, cette progression est habituellement passagère et le risque de progression à long terme ne semble pas s'accroître en raison de la grossesse ${ }^{44}$; on devrait en informer les femmes diabétiques enceintes. Les facteurs qui accroissent le risque de la progression passagère de la rétinopathie durant la grossesse incluent: gravité de la rétinopathie initiale, niveau du contrôle glycémique (que révèlent les niveaux d'hémoglobine glycosylée, A1c), durée du diabète et hypertension concomitante. L'état des femmes enceintes souffrant d'une rétinopathie diabétique non proliférante légère à modérée est rarement permanent, à moins qu'il y ait une hypertension chronique ou une hypertension due à la grossesse $e^{45}$. Lle mécanisme sousjacent à la progression lente de la rétinopathie diabétique durant la grossesse serait lié à l'accroissement de la sécrétion induite de progestérone du facteur de croissance vasculaire endothélial et du flot sanguin rétinien modifié. Si la rétinopathie progresse vers une RDNP ou un OMDCS sévère, l'optométriste devrait prévoir une 


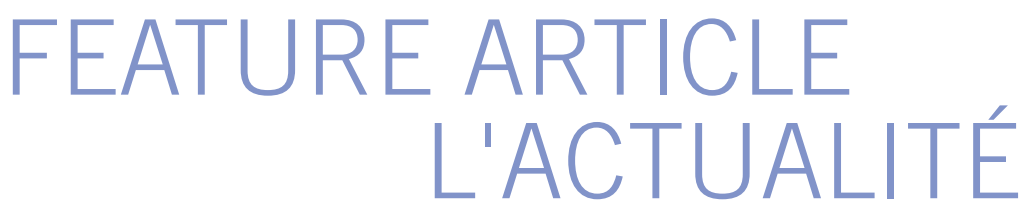

consultation d'urgence avec un spécialiste de la rétine. Dans une perspective de prévention, il faut suivre attentivement l'état de la rétinopathie pendant la grossesse, au moins à chaque trimestre. Les femmes qui envisagent une grossesse malgré des niveaux de rétinopathie sévères devraient d'abord recevoir un traitement de photocoagulation complète de la rétine; environ 30\% des femmes ayant une RDNP modérée développeront une rétinopathie diabétique proliférante pendant la grossesse, par comparaison à seulement $6 \%$ des femmes ayant une RDNP légère ${ }^{46}$.

\section{Manifestations cliniques de la RDNP}

La RDNP légère à modérée se caractérise par la présence de micro-anévrismes, d'hémorragies intrarétiniennes, d'exsudats durs, d'un œdème maculaire diabétique $(\mathrm{OMD})$ et d'anomalies de la zone avasculaire fovéale (ZAF).

Les micro-anévrismes signifient qu'il y a soit un affaiblissement et une dilatation localisés de la paroi capillaire (à la suite d'une perte de péricytes), soit une réponse cellulaire proliférante à une hypoxie focale. Les microanévrismes dont l'apparition s'avère un signe relativement précoce de la rétinopathie diabétique surviennent sur les couches capillaires rétiniennes. La taille des micro-anévrismes est de 12 à 100 micromètres, même si les micro-anévrismes de moins de 30 micromètres ne sont pas visibles à l'ophtalmoscope. À l'opposé des hémorragies, les micro-anévrismes sont ronds, à bords lisses et présentent un réflexe distinct à la lumière centrale. L'occlusion et l'hyalinisation des micro-anévrismes surviendront tôt ou tard.

Les hémorragies intrarétiniennes sont un signe de rupture d'un micro-anévrisme, d'un capillaire ou d'une veinule. Elles épousent une forme qui indiquera leur profondeur dans la rétine; les hémorragies dans les couches rétiniennes plus profondes (couche plexiforme externe/couche nucléaire interne) apparaissent comme des points à bordures distinctes et comme des taches à bordures indistinctes; si elles surviennent dans la couche de la fibre nerveuse rétinienne superficielle, elles apparaissent comme des hémorragies en flammèches. On peut distinguer les hémorragies en points des microanévrismes grâce à l'angiographie à la fluorescéine, même si cela n'est pas une indication clinique pour l'angiographie. Les taches de Roth sont des hémorragies dont le centre est blanchâtre. Elles indiquent la présence d'une auto-occlusion si la source est un micro-anévrisme ou l'accumulation de plaquettes ou de fibrine. Les hémorragies se résorbent généralement à l'intérieur de trois ou quatre mois et n'affectent la vision que si elles sont situées dans la fovéa.

Cliniquement, les exsudats durs ont une apparence jaunâtre brillante ou cireuse. Ils sont souvent situés à l'intérieur ou près de la macula et ils sont fortement associés à un œdème rétinien. Les exsudats durs délimitent souvent une région œéémateuse de la rétine et peuvent être centrés sur un micro-anévrisme exsudatif, auquel cas ils forment souvent une couronne circinée. Les exsudats durs sont des lipoprotéines sériques qui ont traversé les couches capillaires anormalement perméables et qui sont généralement réabsorbées (spontanément ou comme suite à un traitement au laser) à la suite d'une mobilisation des macrophages.

Cliniquement, l'OMD se définit comme un épaississement rétinien que révèle la biomicroscopie stéréoscopique du fond de l'œil. Il se présente comme une accumulation anormale de liquide intrarétinien causée par l'effondrement de la barrière hématorétinienne. En réalité, l'OMD présente un ensemble de signes contenus dans l'expression de la maculopathie diabétique, nommément l'œdème, les exsudats durs et les changements ischémiques. Comme il fallait s'y attendre, la présence d'un OMD est fortement associée aux exsudats durs et aux micro-anévrismes. L'OMD peut survenir relativement tôt dans le développement de la rétinopathie diabétique.

Les anomalies de la ZAF sont des zones capillaires intrarétiniennes non perfusées entourant la fovéa, visibles grâce à l'angiographie. Physiologiquement, les capillaires remplis de fluorescéine entourent mais n'empiètent pas sur la fovéa de couleur sombre; cela crée une ZAF d'un diamètre de 350 à $1000 \mu \mathrm{m}$. Les anomalies de la ZAF associées à la rétinopathie diabétique incluent: bordures irrégulières, bourgeonnement des capillaires dans la ZAF, agrandissement des espaces intercapillaires entourant la fovéa (c.-à-d. occlusion des capillaires), formation de nouveaux vaisseaux sanguins, agrandissement du di-

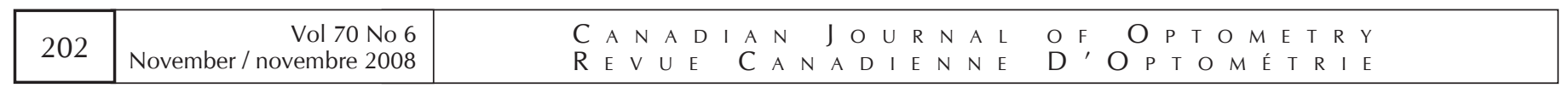




\section{L'ACTUALITÉ FEATURE ARTICLE}

amètre de la ZAF et dilatation des capillaires existants. En dernier ressort, la perte de perfusion du réseau capillaire entourant la ZAF provoquera une perte de vision. C'est pourquoi une perte de vision inexpliquée chez un patient diabétique peut être le signe d'une maculopathie diabétique ischémique nécessitant un aiguillage rapide chez un spécialiste rétinien et une angiographie.

Une RDNP de modérée à sévère se caractérise par la présence d'exsudats cotonneux, de nodules veineux et d'anomalies microvasculaires intrarétiniennes (AMIR). Ces signes rétiniens annoncent les changements morphologiques à venir associés à la rétinopathie diabétique, progressant jusqu'à l'occlusion et la fuite vasculaires. Sans grande surprise, on croit que l'occlusion artériolaire plutôt que l'occlusion capillaire cause une ischémie plus sévère, ce que révèlent particulièrement les exsudats cotonneux, les nodules veineux et les hémorragies intrarétiniennes plus nombreuses.

Cliniquement, les exsudats cotonneux, quelquefois appelés exsudats mous, sont des lésions striées aux bordures cannelées, duveteuses, d'un blanc jaunâtre. Ce sont des zones d'infarctus localisé, d'où perte d'apport sanguin sur la couche de fibres nerveuses et obstruction du flux axoplasmique dans les axones des cellules ganglionnaires et une inflammation des fibres nerveuses. L'agression ischémique endommagera la couche des fibres nerveuses puisque les fibres nerveuses intrarétiniennes sont atrophiées. Les exsudats cotonneux sont souvent accompagnés d'hémorragies en taches sombres qui, croit-on, indiquent une occlusion artériolaire partielle, ou une occlusion complète suivie d'une reperfusion. Les exsudats cotonneux et les hémorragies en taches sombres sont donc généralement le signe de la présence de capillaires non perfusés, mais quelques exsudats cotonneux peuvent survenir tôt dans la rétinopathie diabétique ou de façon passagère après l'amorce d'un contrôle glycémique strict.

Cliniquement, les nodules veineux sont un rétrécissement focal ou une dilatation focale des veinules rétiniennes. Les nodules veineux sont dus à l'épaississement et à la dégénérescence hyaline de la paroi veineuse. Les autres anomalies veineuses incluent: formation de «boucles», reduplication de segments, formation d'une gaine veineuse et rétrécissement focal. Les anomalies veineus- es en chapelet sont adjacentes aux capillaires non perfusés et sont souvent associées aux exsudats cotonneux.

Cliniquement, les anomalies microvasculaires intrarétiniennes (AMIR) se révèlent comme des dilatations irrégulières segmentées, des canaux de dérivation des capillaires tortueux (shunts) entre les artérioles et les veinules. Les AMIR se situent près des territoires des capillaires non perfusés et peuvent être le signe d'une néovascularisation intrarétinienne ou d'une dilatation des capillaires préexistants.

Les anomalies veineuses en chapelet, les AMIR et les hémorragies intrarétiniennes, spécialement les hémorragies en taches sombres à bordures indistinctes, sont de bons indicateurs d'ischémie rétinienne. Ils annoncent une progression vers la $\mathrm{RDP}^{47}$. On croit que la présence d'exsudats cotonneux (exsudats mous) est le signe de changements ischémiques, mais l'acuité mesurée par ETDRS démontre qu'ils ne sont pas des signes fiables de la progression de la RD.

Causes de la perte de la vue chez les personnes atteintes de rétinopathie diabétique: œdème maculaire diabétique cliniquement significatif

L'œdème maculaire diabétique cliniquement significatif (OMDCS) signifie que la fovéa, et donc l'acuité visuelle, est menacée. L'une des principales causes des handicaps visuels et de la cécité chez les diabétiques demeure l'OMDCS et il peut survenir à n'importe quel stade de la rétinopathie diabétique. A partir d'une évaluation clinique par biomicroscopie stéréoscopique du fond de l'œil, la «Early Treatment of Diabetic Retinopatby Study $»^{48}$ définit l'OMDCS, comme étant caractérisé par : 1) tout épaississement rétinien situé à moins de 500 micromètres du centre de la macula; 2) tout épaississement rétinien d'au moins un diamètre papillaire de taille et situé à au plus un diamètre papillaire du centre de la macula ou, 3) la présence d'exsudats durs situés à moins de 500 micromètres du centre de la macula avec épaississement rétinien adjacent. L'œdème maculaire peut être focal ou diffus. L'œdème maculaire focal résulte d'une fuite des microanévrismes ou des AMIR et, cliniquement, sont des territoires localisés d'épaississement rétinien souvent entourés d'un anneau adipeux. L'œdème maculaire dif- 
fus est un épaississement général de la rétine causé par la fuite généralisée des capillaires situés au pôle postérieur, probablement sous l'épithélium pigmentaire rétinien. L'œdème maculaire diffus est associé à des exsudats lipidiques minimaux. Les résultats des traitements expérimentaux contrôlés, particulièrement ceux de l'ETDRS, ont démontré l'importance du traitement par photocoagulation au laser en grille (associé à la coagulation laser focale quand cela est approprié) dans le cas de l'OMD cliniquement significatif. L'ETDRS indique que le taux de perte de vision diminue de $50 \%$ grâce à la photocoagulation au laser dans les territoires de fuite ${ }^{49}$. Plus récemment, on a essayé des médicaments intravitréens anti-VEGF et à base de stéroïdes, spécialement dans les cas d'OMD réfractaires. L'optométriste qui découvre des signes d'un OMDCS devrait diriger le patient en urgence vers un spécialiste rétinien.

Causes de la perte de la vue chez les patients atteints de rétinopathie diabétique:

rétinopathie diabétique proliférante

Par définition, on décrit cliniquement la RDP comme

la croissance de nouveaux vaisseaux sanguins sur la rétine ou sur le disque du nerf optique. Ces vaisseaux prolifèrent le long de la surface rétinienne interne (partie sous-hyaloïdienne) et dans le vitré cortical, avec ou sans composante fibreuse. Typiquement, la néovascularisation survient à 45 ou moins du disque du nerf optique et spécialement sur le disque du nerf optique lui-même. Les nouveaux vaisseaux sanguins situés à une distance d'au plus un diamètre papillaire sont appelés néovascularisation du disque (NVD), tandis que les autres sont appelés néovascularisation d'un autre territoire ("neovascularisation elsewhere », NVE) sur la rétine. La prolifération dans le vitré peut amener des hémorragies sous-hyaloïdiennes, prérétiniennes ou vitréennes et un décollement rétinien tractionnel. Une consultation urgente d'un spécialiste rétinien est recommandée, car la photocoagulation circonscrite ou panrétinienne de la RDP peut diminuer le risque de perte de vision grave de $95 \%$ s'il y a un traitement précoce. Plus récemment, on a démontré que l'utilisation de médicaments intravitréens anti-VEGH comme l'Avastin et le Lucentis a ralenti la progression de la RDP.

\section{Stades de la RDNP et indications} relatives à I'aiguillage

On peut qualifier la RDNP comme non présente, légère à modérée, modérée à sévère, sévère ou très sévère. Les critères d'aiguillage concernant la rétinopathie diabétique varient d'une région à l'autre au Canada, mais les critères suivants peuvent servir de guide.

Tableau clinique de la RDNP légère à modérée : présence de microanévrismes, d'hémorragies intrarétiniennes, d'exsudats durs, d'œdème maculaire diabétique (OMD) et d'anomalies de la zone avasculaire fovéale (ZAF). Généralement, les personnes atteintes de cette forme de RDNP peuvent être suivies annuellement sans danger. On considère souvent cette forme de RDNP comme relativement bénigne, mais comme l'OMDCS peut survenir à n'importe quel stade du développement de la rétinopathie, cela peut porter à confusion. Du point de vue optométrique, l'OMDCS nécessite un aiguillage urgent chez un spécialiste rétinien.

Tableau clinique de la RDNP modérée à sévère: présence d'exsudats cotonneux, de nodules veineux et d'anomalies microvasculaires intrarétiniennes, et signes d'une RDNP légère à modérée. Les personnes atteintes de cette forme de RDNP doivent être examinées par leur optométriste tous les six mois selon la sévérité de cette forme et la présence d'autres comorbidités. Cette forme de RDNP qui indique la présence d'une ischémie rétinienne plus sérieuse devrait être traitée avec soin.

Tableau clinique de la RDNP sévère et très sévère: nombreuses AMIR, exsudats cotonneux, modifications du système veineux spécialement les anomalies veineuses en chapelet. La RDP sévère se caractérise par n'importe lequel des quatre signes suivants («règle du 4-2-1») : hémorragies intrarétiniennes sévères dans les quatre quadrants; nodules veineux dans deux quadrants; AMIR modérément sévères dans un quadrant. Deux des critères susmentionnés constituent une RDP très sévères. Les optométristes devraient rapidement aiguiller le patient chez un spécialiste rétinien, car les RDNP sévères et très sévères annoncent l'imminence d'une RDP et nécessitent souvent une chirurgie au laser préventive.

\section{Recommandations et conclusions}

En tant que fournisseurs de soins oculovisuels primaires, les optométristes doivent jouer un rôle actif dans la ges-

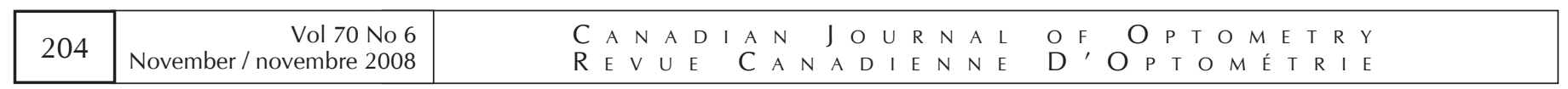




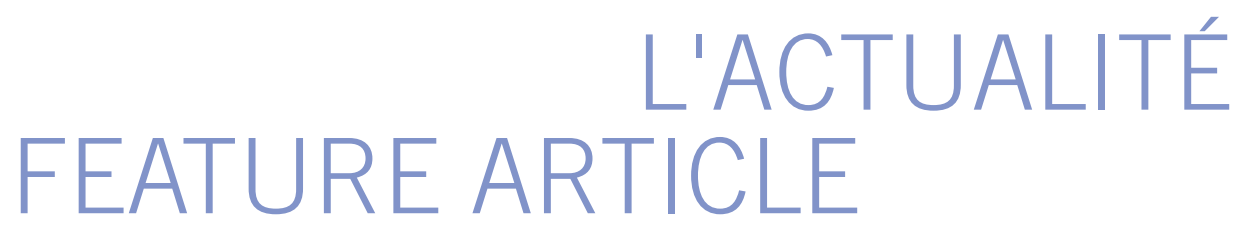

tion, le soin et le traitement des patients diabétiques. Très souvent, nous traitons ces patients pour d'autres conditions oculovisuelles. Souvent nos patients actuels qui développent le diabète ont besoin de conseils sur la façon de gérer les complications possibles, spécialement les complications oculaires. Les optométristes devraient interroger leurs patients diabétiques au sujet de leur taux d'hémoglobine glycosylée (A1c), leur tension artérielle et leur cholestérol. L'Association canadienne du diabète recommande aux diabétiques les niveaux suivants : 7,0\% ou moins pour l'A1c (mais elle reconnaît que cela n'est pas toujours facile spécialement pour les personnes sujettes à des épisodes hypoglycémiques); 130/80 mmHg ou moins pour la tension artérielle; $2,0 \mathrm{mmol} / \mathrm{L} \mathrm{ou}$ moins pour le cholestérol des lipoprotéines de basse densité, et moins de 4,0 pour le cholestérol total des lipoprotéines de haute densité. En tant qu'optométristes, nous devrions conseiller à nos patients diabétiques de modifier leur style de vie, par exemple faire régulièrement de l'exercice et arrêter de fumer. Il est important que nous communiquions les résultats de nos tests à nos patients et aussi à d'autres membres de l'équipe des soins de santé diabétiques afin qu'ils soient pris en charge de façon adéquate et efficiente et qu'ils soient aiguillés vers des soins qui dépassent peut-être notre compétence professionnelle. En nous basant sur les lignes directrices de pratique clinique de l'Association canadienne du diabète de 2003, nous faisons les recommandations supplémentaires suivantes sur la fréquence des examens:

Pour les patients diabétiques de type 1:

Examen au moment du diagnostic puis, cinq ans après l'apparition du diabète, sur une base annuelle chez les personnes de 15 ans et plus.

\section{Chez les patientes diabétiques de type 1}

qui envisagent une grossesse:

Examen avant la grossesse, pendant le premier trimestre et chaque trimestre par la suite, au besoin tout au long de la grossesse et pendant la première année après l'accouchement.

Chez les patients diabétiques de type 2 :

Examen au moment du diagnostic. La fréquence des examens de suivi devrait être fixée en fonction de la sévérité de la rétinopathie. Chez les patients avec rétinopa- thie minimale ou sans rétinopathie, on recommande un examen une fois l'an ou à tous les deux ans.

Le graphique suivant devrait être utilisé par les optométristes pour gérer les patients diabétiques et pour communiquer leurs résultats aux autres membres de l'équipe de soins de santé diabétiques.

\section{PRISE EN CHARGE OCULAIRE DES PATIENTS DIABÉTIQUES}

\begin{tabular}{|c|c|c|}
\hline $\begin{array}{c}\text { Rapport au médecin } \\
\text { de famille et à }\end{array}$ & $\begin{array}{c}\text { Consultation du Patient } \\
\text { avec son médecin de famille }\end{array}$ & \multirow{2}{*}{$\begin{array}{c}\text { Rapport au médecin } \\
\text { de famille et à } \\
\text { l'équipe des soins } \\
\text { de santé diabétique }\end{array}$} \\
\hline $\begin{array}{l}\text { T'équipe des soıns } \\
\text { de santé diabétique }\end{array}$ & Diagnostic du Diabète & \\
\hline Présence de & Référence & \multirow{2}{*}{ 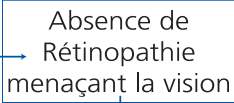 } \\
\hline $\begin{array}{c}\text { Rétinopathie } \\
\text { menaçąnt la vision }\end{array}$ & $\begin{array}{c}\text { Examen par un } \\
\text { Optométriste ou un } \\
\text { Ophtalmologiste }\end{array}$ & \\
\hline $\begin{array}{l}\text { Reférence à un } \\
\text { Spécialiste de rétine } \\
\text { / Ophthalmologiste }\end{array}$ & Ophtalmologiste & \\
\hline $\begin{array}{l}\text { Examen, } \\
\text { Traitement } \\
\text { et suivi requis }\end{array}$ & $\begin{array}{c}\text { Rapport, référence } \\
\text { pour des soins continus }\end{array}$ & $\begin{array}{c}\text { Traitement et suivi } \\
\text { dans le cadres } \\
\text { de la Practique }\end{array}$ \\
\hline
\end{tabular}

Note : L'équipe de soins de santé diabétiques fait référence à l'équipe de base qui inclut le médecin (de famille et/ou spécialiste) et les éducateurs des diabétiques (infirmière et diététiste).

\section{References}

1. Canadian Diabetes Association (2003). In Diabetes Facts. http:// www.diabetes.ca/Section_About/FactsIndex.asp

2. Amos AF, McCarty DJ and Zimmet P (1997). The rising global burden of diabetes and its complications: estimates and projections for the year 2010. Diabetic Med 14 (Suppl 5): S1-S85.

3. King H, Aubert R and Herman W (1998). Global burden of diabetes, 1995 - 2025. Prevalence, numerical estimates and projections. Diabetes Care 21: 1414-1431.

4. Dannenbaum D, Kuzmina E, Lejeune P, Torrie J Gangbe M (2008). Prevalence of diabetes and diabetes-related complications in First Nations communities in Northern Quebec (Eeyou Istchee), Canada. Can J Diabetes 32: 46-52.

5. Zimmet P (2000). Globalization, coca-colonization and the chronic disease epidemic: can the doomsday scenario be averted? J Intern Med 247: 301-310.

6. Time Magazine (2003). Diabetes. Are you at risk? Canadian Edition, Dec 8th, 2003.

7. Canadian Diabetes Association website http://www.diabetes.ca/ Section_About/prevalence.asp

8. Whitaker NA (2002). Diabetes Mellitus: A Systemic Review and Update. Practical Optometry 13: 5.

9. Health Canada (2003). Diabetes in Canada. 2nd Edition. Chapter 4. Health Outcomes. http://www.phac-aspc.gc.ca/publicat/dicdac2/english/05contents_e.html

10. Health Canada (2003). In Diabetes in Canada. 2nd Edition. Chapter 5. Use of Health Services and Costs. http://www.phacaspc.gc.ca/publicat/dic-dac2/english/05contents_e.html

\section{A N A D I A N J O U R N A L O F O P T O M E T R Y \\ REVUE C A N A D I E N N E D'O P T O M É T R I E}

Vol 70 No 6

November / novembre 2008 
11. American Diabetes Association (2003). Clinical Practice Recommendations 2003. Implications of the United Kingdom prospective diabetes study. Diabetes Care 26 (supplement 1): S28-S32.

12. Canadian Ophthalmological Society (2002). Submission to the Commission on the Future of Health Care in Canada; June 2002.

13. Diabetes in Canada Evaluation (DICE) (2005). Release, Canadian Diabetes Association. September 2005. http://www.gsk.ca/ english/docs-pdf/20050920.pdf

14. Zimmet PZ, Tuomi T, Mackay IR et al (1994). Latent autoimmune diabetes mellitus in adults (LADA): the role of antibodies to glutamic acid decarboxylase in diagnosis and prediction of insulin dependency. Diabet Med 11: 299-303.

15. Pacaud D, Bourgh S (2004). The Family Physician and Diabetes in Childhood and Adolescence; Canadian Diabetes 17 (3). Autumn 2004.

16. Krumholtz, David M (2005). An Overview of Diabetes for the Primary Care Optometrist, Clinical and Refractive Optometry 16 (11).

17. Pacaud D, Meltzer S (2005). An Ounce of Prevention is Worth a Pound of Cure, Canadian Diabetes Volume 18 (4) Winter 2005.

18.Glaser N, Barnett P, McCaslin I, et al (2001). Risk factors for cerebral edema in children with diabetic ketocaidosis. New England Journal of Medicine 344: 264-269

19. Lawerence SE, Cummings, EA, Galboury I, et al (2005). Population based study of incidence and risk factors for cerebral edema in pediatric ketoacidosis, Journal of Peadritics 146: 688692.

20. Atkinson MA and Eisenbarth GS (2001). Seminar. Type 1 diabetes: New perspectives on disease pathogenesis and treatment. The Lancet: 358 (9277): 221-229.

21. Javitt, JC, Canner, JK, Sommer, A (1989). Cost effectiveness of current approaches to the control of retinopathy in type I diabetes. Ophthalmology 96: 255-264

22. Klein R, Klein BEK, Moss SE et al (1989). The Wisconsin epidemiologic study of diabetic retinopathy, IX: Four-tear incidence and progression of diabetic retinopathy when age at diagnosis is less than 30 years; Arch Ophthalmol 107: 237-243

23. Klein R., Klein BEK, Moss SE., et al (1989). The Wisconsin epidemiologic study of diabetic retinopathy. X : Four-year incidence and progression of diabetic retinopathy when age at diagnosis is 30 years or more. Arch Ophthalmol 107: 244-249.

24. Klein R., Klein BEK., Moss SE., et al (1994). The Wisconsin Epidemiologic study of diabetic retinopathy.XIV: Ten-year incidence and progression of diabetic retinopathy. Arch Ophthalmol 112: 1217-1228.

25. McIntosh D, Kjernisted K, Hammond J (2008). Diabetes and depression: What is the association between these common, chronic illnesses? Canadian Diabetes 21 (1): 3-7.

26. Voruganti LP, Parker G (2008). Diabetes and mental health: New frontiers, challenges and opportunities. Canadian Diabetes 21 (1): 8-9.

27. Bohlman H (2006). The obesity epidemic: A New Role for O.D.s? Review of Optometry 2006, September.

28. Pacaud, D, Nabata L (2007). Children Learn by Example; Stemming the Tide of Childhood Obesity.
Canadian Diabetes 20 (2).

29. Skorin L (2003). Diabetes Diagnosis. Review of Optometry, April 2003.

30. Cavallerano JD (1994). The Myriad Threats of Diabetes. Review of Optometry 1994, October.

31. Jovanovic L 2003). Gestational Diabetes Mellitus: The Case for Euglycemia. Canadian Journal of Diabetes 27(4): 428-432.

32. Ludwig S (2003). Risks to Children of Women With Diabetes: Fact or Myth? Canadian Diabetes 16 (4) Winter 2003.

33. Peterella R., Ludwig S (2003). Management of Obesity; Linking Lifestyle Behaviors to Targeted Medication Adjuncts. Canadian Diabetes 16 (1). Spring 2003.

34. Bowman AB., Foster C (2007). Physical Activities Advisement Practices in Diabetes Education Centers across Canada. Canadian Journal of Diabetes 31(2): 131-139.

35. Foot, DK (1996). Boom, Bust and Echo: How to Profit from the Coming Demographic Shift. Pubs; Macfarlane Walter and Ross.

36. CNIB (2001). The Canadian National Institute for the Blind Submission to the Commission on the Future of Health Care in Canada. www.cnib.ca/eng/publications/health_care.

37. HALS (1994): Health and Activity Limitations (HALS) 1991 postcensual disability survey (released 1994): Statistics Canada. www. statcan.ca/english/sdds/3252.

38. Davis J (1996). The Ocular Manifestations of Diabetes, Practical Optometry 7(3): 84-91.

39. Ontario Ministry of Health and Long-Term Care (2004). Diabetes Task Force, Report to the Ministry of Health and Long-Term Care. http://www.health.gov.on.ca/english/public/pub/ ministry_reports/diabetes_taskforce/diabetes_taskforce.html

40. Kahn HA Hiller R (1974). Blindness caused by diabetic retinopathy. Am J Ophthalmol 78: 58-67.

41. Bowyer NK (2003). Diabetic Retinal Changes Linked to Amputation. Review of Optometry 2003, December.

42. Moss SE, Klein R, Klein BEK, Wong TY (2003). Retinal vascular changes and 20-year incidence of lower extremity amputations in a cohort with diabetes. Arch Intern Med 163: 2505-2510.

43. Archer DB (1999). Bowman Lecture 1998. Diabetic retinopathy: some cellular, molecular and therapeutic considerations. Eye 13: 497-523.

44. The Diabetes Control and Complications Trial Research Group (2000). Effect of pregnancy on microvascular complications in the diabetes control and complications trial. Diabetes Care 23: 1084-1091.

45. Klein BE, Moss SE, Klein R (1990). Effect of pregnancy on diabetic retinopathy. Diabetes Care 13: 34-40.

46. Chew EY, Mills JL, Metzger BE et al (1995). Metabolic control and progression of retinopathy. The Diabetes in Early Pregnancy Study. Diabetes Care 18: 631-637.

47. Early Treatment Diabetic Retinopathy Study Research Group (1991). ETDRS Report Number 12. Fundus photographic risk factors for progression of diabetic retinopathy. Ophthalmology 98: 823-833.

48. Early Treatment Diabetic Retinopathy Study Research Group (1987). Treatment techniques and clinical guidelines for photocoagulation of diabetic macular edema. ETDRS Report Number 2. Ophthalmology 94: 761-774.

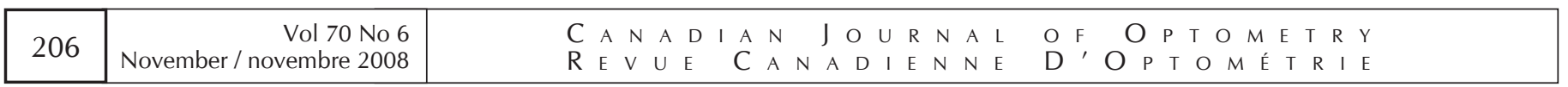

